\title{
Virtual patient cases: a qualitative study of the requirements and perceptions of authors
}

\author{
Nancy Posel ${ }^{1}$, Bruce M. Shore ${ }^{2}$, David Fleiszer ${ }^{1}$ \\ ${ }^{1}$ Faculty of Medicine, McGill University, Canada \\ ${ }^{2}$ Faculty of Education, McGill University, Canada
}

Correspondence: Nancy Posel, The Lady Meredith House, 1110 Pine avenue west, Suite 18, Montreal, QC H3A 1A3, 514

398 2077. Email: nancy.posel@mcgill.ca

Accepted: August 25, 2012

\begin{abstract}
Objectives: To explore the self-defined knowledge requirements of medical educators who had authored virtual patient cases, to examine their perceptions about the actual authoring or development process, and to then compare these responses with available literature on this subject.

Methods: Qualitative data from semi-structured interviews were analysed using an adaptation of constant-comparative methodology. A purposive sample of eight medical educators with expertise in authoring virtual patient cases participated in this study.

Results: Analysis of data revealed five themes: (a) The role of virtual patient cases within medical curricula; (b) The role of curricular revision; (c) The learner; (d) The educator; and (e) Outstanding issues. Case design specifications were also described. Participants emphasized that a comprehen-
\end{abstract}

sive picture of a virtual patient case-based curriculum did not yet exist.

Conclusions: Data from this study defined specific areas of relevance for virtual patient cases creation from the perspective of expert virtual patient case authors. The results demonstrated a general congruence between available academic theory as reported in the literature and the practical realities of virtual patient case authoring. However, specific issues that have not yet been addressed in the literature were also emphasized by the participants and these findings can be used to support medical educators in their development of new virtual patient cases as well as provide direction for research and publications in the this field.

Keywords: Virtual patient cases, virtual patient case authoring, virtual patient case design

\section{Introduction}

This study explored the requirements and perceptions of a purposive sample of eight medical educators who had authored virtual patient cases. Their cases were authored in their individual medical schools and were part of their core curricular teaching commitments. Each participant was considered to have expertise in virtual patient case authoring. Expertise was determined by authorship of a minimum of two virtual patient cases, leadership in the creation of working groups or interest groups that supported the development and utilization of these cases within medical curricula, and academic appointments in their schools in the field of medical informatics with a focus on the use of virtual patient cases.

During the interview process, participants' views about virtual patient case design were also sought. The themes that arose from these data were then compared to current literature on virtual patient case authoring.

The resulting data explicitly identified pertinent issues and challenges faced by the study respondents. The research data can be used to support a better understanding of the challenges faced by medical faculty, many of whom are now increasingly responsible for the development of virtual patient cases. As well, these data will help to determine whether the literature that is currently available pertinent to virtual patient case authoring adequately addressed the requirements of faculty. Identification of issues as yet unaddressed in the literature provides opportunities for future authors interested in contributing to this field; and helps determine directions for potential research and publications in support of new virtual patient case authors. 


\section{Definition and rationalization for virtual patient cases}

Virtual patient cases are "interactive computer simulations of real-life clinical scenarios for the purpose of medical training, education or assessment" ${ }^{\text {. }}$. Virtual patient cases have been described as able to provide realistic nonthreatening clinical scenarios for learning and assessment, ${ }^{1}$ effectively support knowledge transfer, ${ }^{2}$ and present casebased opportunities for problem-based inquiry learning. They are recognized by oversight organizations such as the Liaison Committee on Medical Education (LCME) Accreditation Standards, ${ }^{3-5}$ and are increasingly evident, and progressively more embedded, within core undergraduate medical curricula. ${ }^{6}$

\section{Available literature on virtual patient cases}

Discussion papers and descriptive reviews have rationalized support for virtual patient cases as able to (a) provide learners with necessary diverse, and critical, clinical experiences, ${ }^{7}$ (b) support exponential increases in medical domain knowledge, ${ }^{8}$ (c) address decreased faculty teaching time, ${ }^{7}$ (d) assist schools with distributed campuses where curricula must be accessible to students in geographically disparate areas, ${ }^{4}$ and (e) recognize expectations of digital learners, comfortable with technology.

This same literature is generally optimistic about the value and utility of these cases, noting "compelling instructional capabilities". Authors have suggested that virtual patient cases provided learners with standardized and comprehensive scenarios; supported clinical pattern recognition through deliberate practice; deliver continuous feedback, and formative and summative assessment, ${ }^{10,11}$ permitted clinical analysis, decision-making, hypothesis generation and treatment planning, ${ }^{12,13}$ allowed learners to actively "focus on a standard set of educational competencies"14 and modelled therapeutic communication strategies used by experts. ${ }^{15}$ Within Simulation Centers, when sequenced and blended with standardized patients and physical simulators, they provided contextually relevant models for clinical management. ${ }^{16}$

Fewer empirical studies are available. These have demonstrated effects on knowledge transfer, acquisition, and application..$^{5,7,10}$ Meta-analyses of efficacy and pedagogical impact have revealed positive effects on learning. ${ }^{17,18}$ Most recently, a randomized controlled study found that modules similar to linear virtual patient cases, when integrated with Simulation Center activities, had a significantly positive impact on actual patient clinical outcomes. ${ }^{19}$

No research exists on the individual requirements and perceptions of medical educators responsible for authoring or integrating virtual patients within their teaching practice. No research has attempted to determine whether the available literature on virtual patient cases meets the selfdefined learning needs or requirements of medical educators interested in authoring these cases. Two research questions were addressed:
1. How do you personally author virtual patient cases and can you identify issues that you perceive as relevant to your experiences with virtual patient case authoring and design?

2. How are virtual patient cases currently developed and used in your school?

\section{Methods}

A qualitative, exploratory case research design was chosen for this study based on the study's congruency with characteristics described by Yin. ${ }^{20}$ The study asked medical educators to describe their individual requirements and perceptions about authoring virtual patient cases and to define their case design specifications. Institutional review board approval from McGill University was obtained prior to initiation of the study.

\section{Study design}

Individual semi-structured interviews were conducted with a purposive sample of eight medical educators. The interviews ranged in length from one to three hours. This number is considered adequate for a multiple-case study. ${ }^{20,21}$ Interview questions were developed by the researchers. Interviews were audiotaped and then independently transcribed.

\section{Sample}

Selected participants were determined to have expertise in virtual patient case authoring. All had authored virtual patient cases and were supportive of their use in medical education. At the time of the study none had a peerreviewed publication on virtual patient cases. Contributions by participants to the literature subsequent to their interviews have not been included in this assessment. Participants were geographically located in Canada and the northeastern United States. They represented six medical schools, three in Canada and three in the United States. All of the participants were medical faculty with a minimum of eight years as academic teaching staff. All study participants signed consents and anonymity was assured. The participants were identified in the research through unique identifiers.

\section{Data collection}

The research was conducted in a natural setting and data collection, in the form of semi-structured interviews, took place in the field, in this case, in the participant's school, in either the office or the laboratory of each participant. Interviews lasted between one and three hours. The researcher entered the world of the participant and did not purposefully manipulate or alter the context.

\section{Data analysis}

Analysis of data was inductive, iterative and interactive. Hypotheses were not established a prior but rather emerged from the data. The researchers were the primary data 
collection instruments, and interacted with each participant individually. The research focused on the participants' perceptions, expertise, and experiences. Realities were constructed by the research participants and the researcher reported on these realities and relied on the participant's voices and interpretations of virtual patient case authoring. An adaptation of constant comparative inquiry methodology was used. This adapted methodology allowed themes to be noted even if they were common only to a subset of the participants. Data were compared and contrasted and then constructed into themes. Development of themes required repeated reviews of the transcripts. During the initial coarse-grained phase, the circumstances of the interview and the actual words of the participant were examined as transcribed. Data were then placed in distinct units of analysis and decontextualized. This process required close readings and rereadings of the material, reflection, and the development of an iterative series of transcript documentations. It permitted the researchers to make comparisons across individual participants' associations among participants' responses, and assignments of codes. In parallel, rules of inclusion were developed by the researchers to determine specific requirements that needed to be fulfilled for data categorization to occur. These rules provided a system for classifying the material, and allowed the researchers to decide whether or not a portion of data fit a certain category. They were based on what was contained in the data, not on researcher abstractions and continued until the categories were saturated. In this way, unanticipated themes emerged.

In summary data analysis was based on an adaptation of constant-comparative methodology. It was inductive, iterative, and emergent, and led to participant-defined themes and subthemes. Themes were then compared to the existing literature. Analysis was done by the authors manually.

After a thematic analysis of the participants' responses, the investigators then compared the relationship between these responses with literature currently available on virtual patient cases and virtual patient case authoring.

\section{Results}

\section{Virtual patient case development and utilization}

Five themes emerged, three of which were associated with either one or several subthemes. The themes and subthemes that emerged from the Participant data were:

1. The role of virtual patients in medical curricula; subthemes included (a) their use in individual or group settings, (b) their ability to "prime the pump," and (c) addressing gaps in the matrix of programs due to the loss of the hospital as a clinical laboratory.

2. Curricular revision

3. The learner; subthemes (a) the $21^{\text {st }}$ century learner, (b) the student's hunger for information and integration into the care team, (c) the ability of virtual patient cases to allow learners to view a patient care trajectory from a longitudinal perspective, and (d) integrating humanity and physicianship.

4. The educator

5. Outstanding issues; subthemes (a) a lack of virtual patient case authoring guidelines, (b) the need for evidence and research, (c) nomenclature, and (d) selfreflection as an unexpected consequence of virtual patient case-authoring.

Each theme and subtheme is reported. Subsequently, each theme and subtheme is related, where possible, to the current literature on virtual patients.

\section{The role of virtual patient cases in undergraduate medical curricula}

a) Individual or group settings: participants were mixed in their consideration about whether virtual patient cases were best used individually or in small groups; four supported their use in small group settings, while the others remained uncertain. This lack of clarity could be indicative of a dearth of research and thus evidence about the efficacy of different settings. No mention was made of the use of these cases in whole class teaching. The literature has suggested that, although utilization of virtual patients in either small groups or by students individually does not mitigate the potential of the medium, it may have significant implications on instructional design, ${ }^{4,15}$ and on learning outcomes. ${ }^{10}$ There is support in the literature for the efficacy of virtual patient cases in small groups, ${ }^{22}$ however, there is support for singular use, most particularly within the context of deliberate practice and the development of individualized competencies. $^{5,10,11}$

b) Prime the pump: five of the eight participants suggested students used virtual patient cases to link the basicscience teaching with the reality of clinical encounters. One participant suggested that these cases "primed the pump" (Participant 3) another noted that virtual patient cases "allow students to see the clinical significance of the basic science" (Participant 2). The literature has noted the potential of these cases to support learner integration of the basic sciences with models of clinical practice, ${ }^{23-26}$ to integrate multiple complex knowledge domains into comprehensive practice models, ${ }^{27}$ and to address a post-Flexnerian requirement for an inclusive approach to student teaching. ${ }^{28}$

c) Addressing gaps in the matrix of our programs due to the loss of the hospital as a clinical laboratory: study participants agreed that there are insufficient numbers of inpatients to adequately support medical student learning. Seven of the eight participants stated that virtual patient cases permitted learners' access to necessary but sometimes unavailable clinical situations. Participant 7 remembered when "patients were admitted because they were good teaching cases" and compared this 
to current "patient-turnover pressure". Another participant noted that virtual patients "guarantee that all students experience core mandated clinical scenarios, supplement those that were missed, and address the gaps in the matrix of our programs, ensuring necessary competencies for accreditation" (Participant 4). Participant 8 suggested that virtual patient cases "allowed students to experience variable presentations of cases" and specified domains such as surgery, paediatrics, medicine, obstetrics, and family medicine that would benefit from their inclusion. The literature has addressed the impact of the lack of inpatients as a teaching resource, ${ }^{7,8}$ the utilization of virtual patients to address knowledge gaps, ${ }^{29}$ to ensure more comprehensive learning experiences, ${ }^{12,14,30}$ to support development of core competencies, ${ }^{10}$ to provide otherwise unavailable clinical experiences ${ }^{5}$ and to guarantee availability of standardized material. ${ }^{9}$

\section{Curricular revision}

Inclusion of virtual patient cases within on going curricular revision was addressed by study participants. All of the participants felt that new curricula should include virtual patients, and highlighted their utility in distributed learning environments as well as their ability to meet learners' requirements for asynchronous as well as synchronous online learning. This finding is not surprising as participants were chosen for their interest in virtual patient cases.

The literature highlighted the role of virtual patients in medical curricula, and emphasized their potential for "termless" learning, ${ }^{31}$ the evolution of informatics-based education, inclusive of virtual patients ${ }^{9,24,32}$ and the utility of virtual patient cases in core undergraduate medical curricula. $^{6}$

\section{The learners}

Six of the study respondents described medical students as expert learners, but as "adolescent rather than adult learners, younger than they were ten years ago" (Participant 7). Three study participants emphasized the need to support professional socialization and as well as domain learning. Interestingly, comfort and supposed proficiency with new technologies were not specifically addressed.

The literature has suggested that the $21^{\text {st }}$ century learner is more technologically adept, has expectations of the accessibility of online material and resources, and uses and presumes that comprehensive and effective technologies are available.

a) The student's hunger for information and integration into the care team: five of eight study participants highlighted their students' hunger for information and their need to be more fully integrated into the healthcare team. They noted "students struggle to find pertinent information and direction during their preclerkship learning" (Participant 2) and described learner frustration associated with advances in medical technology.
One participant suggested that laparoscopic surgeries had resulted in a less active participation, at a very junior level, in operative procedures. He stated that "since cholecystectomy is now done using a laparoscope, the students only see the procedure on a video screen and are less interested in going to the operating room" (Participant 4). This was not an issue addressed in the literature, although, in retrospect, it is an understandable finding.

b) The ability to view a patient care trajectory from a longitudinal perspective: similarly, study participants noted the frustration of students who were unable to follow patients throughout a complete trajectory of longitudinal care. Participants indicated that fewer inpatients and shorter stays had a direct impact on learners' perspectives of a comprehensive trajectory of care. They described a "disconnect" (Participant 4) between learners and patients and worried that this might disenfranchise the medical learner from the patient and impact on affective or emotional components of learning patient care. Respondents stated that "students do not see a natural clinical course" (Participant 2) and felt that virtual patients might permit students to follow patients for "virtual years, learning long-term care management and multisystem care" (Participant 4) suggesting that "these cases can simulate temporal changes in disease as it evolves" (Participant 5). The potential to demonstrate a longitudinal trajectory of care to students was not previously identified in the literature, but highlights a benefit of virtual patient case representations, specifically those that address long term or chronic diseases, or conditions that are longitudinal by their nature.

c) Integrating humanism in practice: five participants agreed that the practice of patient-centred humanistic approaches to care was essential for professional medical socialization. They were concerned that virtual patient cases could potentially divorce students from encounters with real patients, and emphasized that they should be used as adjunctive support, where and when, necessary to "provide relevance and ensure the story of the patient rather than the disease is apparent" (Participant 7).

This perspective is supported by Brawer ${ }^{33}$ who wrote about the importance of developing physicians who, in addition to being scientists, also address the humanistic responsibilities and requirements of their profession. It is also addressed in research that used virtual patients to emphasize empathy rather than problem-solving and the generation of differential diagnoses. ${ }^{15}$

\section{The educator}

Four of the study participants highlighted the lack of clear mandates or support for medical educators interested in developing virtual patient cases. One study participant 
stated "we tend to forget that teachers have been to thousands of lectures, but haven't seen even a handful of virtual patients" (Participant 8) and another noted that "writing cases is hard, it is time consuming and difficult" (Participant 1). All of the study participants recommended an increased role on the part of faculty development. Responses indicated that specific areas for support were related to pedagogy and case authoring. The technology was not highlighted as an issue by any of the respondents.

This lack of support for educators interested in informatics-based modalities such as virtual patients and the potential role of faculty develop to support effective case development was anticipated in the literature. ${ }^{25,26,30,34-36}$

\section{Outstanding Issues}

Several outstanding issues were discussed by the study participants.

a) Lack of authoring guidelines and support for medical educators interested in virtual patient case authoring: five of eight study participants highlighted the lack of guidelines, standards, and standardized processes for virtual patient case development. Respondents noted that guidelines were not readily accessible, and, when available, not comprehensive. These comments are supported in the literature ${ }^{2,4,12,22,37}$ although a recent study ${ }^{38}$ highlighted the efficacy of two specific virtual patient case-authoring methodologies. ${ }^{39,40}$ Thus the extent to which the existing literature can inform virtual patient case authoring may be consistent with virtual patient case authors needs, but insufficient.

b) The need for evidence and research: all eight study respondents worked in highly academic environments and noted the negative impact of a lack of research, evidence, and peer-reviews with respect to virtual patient case utilization. Participants identified a need for "research to accurately describe the efficacy for different virtual patient design approaches" (Participant 1) and "peer-review of cases" (Participant 4). The dearth of research in this field and resulting lack of evidence-based direction have been noted. ${ }^{41-43}$ With respect to virtual patient cases the literature has highlighted a lack of research about the integration of multimedia, ${ }^{44}$ the impact of cognitive load, ${ }^{45}$ strategies for the instructional design of complex learning, ${ }^{37}$ and whether, and how, levels of fidelity in cases impact on learning. ${ }^{46,47}$

c) Nomenclature: five participants remarked that there were no clear criteria to describe virtual patients. Participants felt that "people would argue as to what is a true virtual patient case," (Participant 3 ) and noted that "we need to continue to define and scope the taxonomy of virtual patients," (Participant 8 ) and "develop more precise vocabularies so that we can do research" (Participant 5). This lack of clarity was identified in the literature. ${ }^{4}$ d) Self-reflection as an unexpected consequence of caseauthoring: four of eight study participants highlighted that authoring virtual patient cases had led them to reflect on their own personal journeys as medical educators. Participant 4 stated that "you are putting yourself out there and you could be judged." This was particularly true as publication of virtual patient cases does not require the rigorous peer review associated with journal publications. The impact of case-authoring on selfreflection was unanticipated and no studies on the subject have been reported.

\section{Virtual patient case design}

Participants suggested that case design be addressed prior to case authoring and include (a) a storyboard, (b) determination of content, described as a "good story" (Participant 4) and purpose, (c) alignment of complexity with the level of the learners, learners' expectations, and measurable goals and objectives, (d) exploration of how active learning and interactivity could be integrated within the case, and (e) the need to include formative as well as summative assessment and feedback within the body of the case. Very few of these elements had been previously identified in the literature.

a) Storyboarding: participant 3 noted that "when storyboarding was not used the instructional design was not good". Participant 1 described the process as: "I organized the important elements in a concept map. Then we set up a schema and that was the storyboard". Content and the importance of the story: study participants stressed the importance of a story to help learners "remember and integrate information" (Participant 7). They suggested that authors "start with a real patient to give the case a logical coherence and richness that cannot be invented" (Participant 8). Respondents recommended that authors use naturalistic language and inclusion of the patient's voice.

b) Aligning the case complexity with the level of the learner: all eight respondents emphasized that authors must identify the level of the learner, learner expectations, and determine associated measurable, specific, and realistic goals, objectives, case complexity, and purpose. Respondents suggested that case authors "think who the target audience is" (Participant 4) as well as "who is going to teach it, where, and how" (Participant 5).

c) Case design and repurposing: seven of eight study participants recommended building cases to permit repurposing and noted that the ability to repurposed cases for other target learners ensured a more efficient use of resources. One participant suggested that using this approach so that "you can modify existing content and adjust it for different learners" (Participant 5).

d) Integrating assessment and feedback: four of the study participants highlighted the importance of integrating 
assessment, in some format, as well as feedback within their virtual patient cases.

e) The importance of continuous feedback and formative assessment is integral to literature on pedagogy. ${ }^{9,11}$

f) Integrating active learning and interactivity in virtual patient cases: six of the eight respondents noted the importance of interactivity in their cases. They suggested that virtual patient cases enhance interactive learning and provide opportunities for active decision making. Participants noted that "the students have to make a decision and then deal with the consequences" (Participant 5) and case design should include "multiple points where the learner can reflect on their clinical reasoning processes and form a differential diagnosis" (Participant $6)$.

g) The association of interactivity with cognitive theories specifically the Animation and Interactivity Principles, ${ }^{48}$ the Guided Discovery Principle ${ }^{49}$ and work on the use of multimedia in medical education ${ }^{50}$ was not mentioned by the study Participants.

\section{Discussion}

The first research question examined participants' general perceptions about virtual patient cases. A review of participants' data confirmed that items emphasized by the study participants were available in the literature and that this literature was relevant, accurately represented the learning requirements and experiences of the medical educators who participated in the study, and, demonstrated a clear relationship between current academic theory and the practical realities of medical teaching.

Respondents generally agreed with the potential of virtual patient cases as identified in the literature, ${ }^{7,12,22,29}$ as well as with suggestions that a paradigm shift to a more blended approach might be necessary. ${ }^{5,10,23,24,26,51}$ However, they also emphasized that a comprehensive picture of a virtual patient case-based curriculum had yet to be developed.

They also identified barriers to virtual patient caseauthoring. These included the need for greater support from faculty development services, recognition of their efforts, a lack of authoring guidelines, and a dearth of empirical research. Lack of support for faculty development had been raised by several authors, ${ }^{25,26,30,34,35,36,39}$ as had the need for greater recognition for faculty involved in informatics-based initiatives, ${ }^{2,12,22,37}$ for case-authoring guidelines ${ }^{4}$ and for more research. ${ }^{41-43}$ Study Participants also underscored three items not addressed in the literature: the value of longitudinal cases, the students' hunger to feel more integrated within the clinical care team, and the impact of the process of caseauthoring on medical educators.

Individual approaches to virtual patient case design were discussed. The dearth of research and an evidencebased framework for case utilization and design was addressed by all of the study participants. This issue and the requirement for empirical research were also noted in the literature.

Medical educators agreed generally with academic theoreticians that judicious use of technology in education, and specifically virtual patient cases, supported medical teaching and learning, although they felt that their use needed to be extended and utilized within a curricular transformation that was core to teaching practice rather than perceived as an adjunct or optional.

When virtual patient case authors were interviewed indepth about their requirements and perceptions, we learn more than what could have been obtained solely from a review of the literature for this topic. The literature is consistent with their requirements, but it is not yet sufficient to meet them. This study points to the information that remains missing.

This study demonstrated alignment among the descriptive and prescriptive literature on virtual patient cases, the small number of empirical studies that exist to date, and educators who authored virtual patient cases. It revealed new insights that have not been previously been addressed in the literature. This study contributes toward a researchsupported model for the authoring, design and use of virtual patient cases in medical education.

\section{Limitations of the study}

As well as purposive, the sample size of eight was small, although congruent with the literature for this type of study design. Including more participants might have permitted more extensive perspectives, but convergence was high in the responses. Interviews and activities with faculty less interested in this field, or who were new to the potential and the technology, might have yielded different results, but would likely not have yielded the subtle insights obtained from this sample. Working with less expert samples is a worthy concern of future research, but does not diminish the importance of the information gleaned in this instance.

Additional probes could have been added to the questions for the semi-structured interviews. Data related to specific resistances encountered by the participants, personal experiences of unintended negative outcomes, and attempts by participants to measure the impact of their virtual patient cases, may have resulted in a richer discourse.

The study might have benefited from broader geographic diversity. Academically, all of the participants were associated with large and well-known medical schools. Further research might investigate whether smaller schools, with fewer educators and students, faced similar challenges. All of the involved medical schools were well established. Newer schools may prove to be more accepting of innovative technologies. The study was confined to medical educators and the use of virtual patient cases in medical education. Research on the use of virtual patient cases in other healthcare professions or within an inter- professional paradigm would serve to further this work. 


\section{Acknowledgements}

Only those specified as authors contributed to this paper. No financial grants or contributions from other colleagues or from any institution were obtained. The study did not have any source of funding. The written permission of each person acknowledged was gained.

\section{Conflict of Interest}

The authors declare that they have no conflict of interest.

\section{References}

1. Ellaway R, Candler C, Greene P, Smothers V. An architectural model for MedBiquitous virtual patients. Baltimore: MedBiquitous; 2006.

2. Grunwald T, Corsbie-Massay C. Guidelines for cognitively efficient multimedia learning tools: educational strategies, cognitive load and interface design. Acad Med. 2006;81:213-223.

3. Liaison Committee on Medical Education: LCME accreditation standards. 2008 [cited 09 September 2012]; Available from: http://www. lcme.org/functionslist.htm.

4. Peterson L, Powell B. On-line eCase authoring tools: for the use of the UBC undergraduate, postgraduate, continuing professional development knowledge translation and interprofessional education programs: a vision and scope of the project and a comparative review. 2007; University of British Columbia, Faculty of Medicine, internal discussion paper,1-29.

5. Tworek J, Coderre S, Wright B, McLaughlin K. Virtual patients: ED2 band-aid or valuable asset in the learning portfolio? Acad Med. 2010;85:155158.

6. Poulton T, Balasubramaniam C. Virtual patients: a year of change. Med Teach. 2011;33:933-937.

7. Kalet A, Coady S, Hopkins M, Hochberg M, Riles T. Preliminary evaluation of the web initiative for surgical education (WISE-MD). Am J Surg. 2006;194:89-93.

8. Saunders F. Building the cyber medical school. NYU Physician. Winter 2004; 48-50.

9. AAMC. Effective use of educational technology in medical education colloquium on educational technology: recommendations and guidelines for medical educators. Washington: Association of American Medical Colleges; 2007.

10. Cook DA, Triola MM. Virtual patients: a critical literature review and proposed next steps. Med Educ. 2009;43:303-311.

11. Ericsson KA. Deliberate practice and the acquisition and maintenance of expert performance in medicine and related domains. Acad Med. 2004;79:S70-S81

12. Kamin C, O'Sullivan P, Deterding R, Younger M. A comparison of critical thinking in groups of third-year medical students in text, video and virtual PBL case modalities. Acad Med. 2003;78:204-211.

13. Srinivasan M, Wilkes M, Stevenson F, Nguyen T, Slavin S. Comparing problem-based learning with case-based learning: effects of a major curricular shift at two institutions. Acad Med. 2007;82:74-82.

14. Leong SL, Baldwin CD, Adelman AM. Integrating web-based computer cases into a required clerkship: development and education. Acad Med. 2003;78:295-301.

15. Bearman M. Is virtual the same as real? Medical students' experiences of a virtual patient. Acad Med. 2003;78:538-545.

16. Bell RH. Presidential address: why Johnny cannot operate. Surgery. 2009;146:533-542.

17. Cook DA, Levinson AJ, Garside S. Time and learning efficiency in Internet-based learning: a systematic review and meta-analysis. Adv Health Sci Educ Theory Pract. 2011;45:227-238.

18. Gesundheit N, Brutlag P, Youngblood P, Gunning WT, Zary N, Fors U. The use of virtual patients to assess the clinical skills and reasoning of medical students: initial insights on student acceptance. Med Teach. 2009;31:739-742

19. Zendejas B, Cook DA, Farley DR. Teaching first or teaching last: does the timing matter in simulation-based surgical scenarios? J Surg Educ. 2010;69:208-214.
20. Yin RK. Case study research: design and methods. Thousand Oaks: Sage; 2003.

21. Padula MA, Miller DL. Understanding graduate women's re-entry experiences: case studies of four psychology doctoral students in a Midwestern university. Psychol Women Q. 1999;23:327-343.

22. Kim S, Phillips WR, Pinsky L, Brock D, Phillips K, Keary J. A conceptual framework for developing teaching cases: a review and synthesis of the literature across disciplines. Med Educ. 2006;40:867-876.

23. Birden H, Page S. $21^{\text {st }}$ century medical education. Aust Health Rev. 2007; 31:341-350

24. Greenhalgh T. Computer assisted learning in undergraduate medical education. BMJ. 2001;322:40-44.

25. Nageswari KS, Malhotra AS, Kapoor N, Kaur G. Pedagogical effectiveness of innovative teaching methods initiated at the department of physiology, government medical college, Chandigarh. Adv Physiol Educ. 2004;28:51-58.

26. Ruiz J, Mintzer M, Leipzig R. The impact of e-learning in medical education. Acad Med. 2006;8:207-212.

27. van Merrienboer JG, Clark RE, de Croock MBM. Blueprints for complex learning: The 4C/ID-Model. Educational Technology Research and Development. 2002;50:39-61.

28. Irby DM, Cooke M, O’Brien BC. Calls for reform of medical education by the Carnegie foundation for the advancement of teaching: 1910 and 2010. Acad Med. 2010;85:220-227.

29. Cook D, McDonald FS. E-learning: is there anything special about the "e"? Perspect Biol Med. 2008;51:5-21.

30. Lujan HL, DiCarlo SE. First-year medical students prefer multiple learning styles. Adv Physiol Educ. 2006;30:13-16.

31. Koschmann T. Medical education and computer literacy: learning about, through and with computers. Acad Med. 1995;70:818-821.

32. Huang G, Reynolds R, Candler C. Virtual patient simulation at U.S. and Canadian medical schools. Acad Med. 2007;82:446-451.

33. Brawer JR. The value of a philosophical perspective in teaching the basic medical sciences. Med Teach.2006;28:472-474.

34. Goldberg HR, Haase E, Shoukas A, Schramm L. Redefining classroom instruction. Adv Physiol Educ. 2006;30:124-127.

35. Michael J. Where's the evidence that active learning works? Adv Physiol Educ. 2006;30:159-167.

36. Silverthorn DU. Teaching and learning in the interactive classroom. Adv Physiol Educ. 2006;30:135-140.

37. van Merrienboer JG, Kester L. The four-component instructional design model: multimedia principles in environments for complex learning. In: Mayer RE, editor. The Cambridge handbook of multimedia learning. New York: Cambridge University Press; 2005. p. 71-96.

38. Patel V, Aggarwal R, Taylor D, Darzi A. Implementation of virtual online patient simulation. Stud Health Technol Inform. 2011;163:440-446. 39. Posel N, Fleiszer D, Shore BM. 12 tips: Guidelines for authoring virtual patient cases. Med Teach. 2009;31:701-709.

40. Triola MM, Campion N, McGee JB, Albright S, Greene P, Smothers V, et al. An XML standard for virtual patients: exchanging case-based simulation in medical education. AMIA Annu Symp Proc. 2007;741-745.

41. Bearman M, Cesnik B, Liddell M. Random comparison of virtual patient models in the context of teaching clinical communication skills. Med Educ. 2001;35:824-832.

42. Chumley-Jones HS, Dobbie A, Alford CL. Web-based learning: sound education method or hype? A review of the evaluation literature. Acad Med. 2002;77:S86-S93.

43. Cook D. The research we still are not doing: an agenda for the study of computer-based learning. Acad Med. 2005;80:541-548.

44. Mayer RE. Introduction to multimedia learning. In: Mayer RE, editor. The Cambridge handbook of multimedia learning. New York: Cambridge University Press; 2005. p. 1-16.

45. Sweller J. Implications of cognitive load theory for multimedia learning. In: Mayer RE, editor. The Cambridge handbook of multimedia learning. New York: Cambridge University Press; 2005. p. 19-30.

46. Mayer RE, Heiser J, Lonn, S. Cognitive constraints on multimedia learning: when presenting more material results in less understanding. Journal of Educational Psychology. 2001;93:187-198. 
47. Matsumoto E, Hamstra S, Radomski S, Cusimano M. The effect of bench model fidelity on endourological skills: a randomized controlled study. J Urol. 2002;167:1243 - 1247.

48. Betrancourt M. The animation and interactivity principles in multimedia learning. In: Mayer RE, editor. The Cambridge handbook of multimedia learning. New York: Cambridge University Press; 2005. p. 287-296.

49. de Jong $\mathrm{T}$. The guided discovery principle in multimedia learning. In:
Mayer RE, editor. The Cambridge handbook of multimedia learning. New York: Cambridge University Press; 2005. p. 215-228.

50. Clark, R. C. Multimedia learning in e-courses. In: Mayer RE, editor. The Cambridge handbook of multimedia learning. New York: Cambridge University Press; 2005. p. 589-616.

51. Friedman CP. The research we should be doing. Acad Med. 1994;69:455 457. 